\title{
Virilization in a postmenopausal woman: ovarian steroid cell tumour
}

\author{
S Pathmanathan ${ }^{1}$, Noel P Somasundaram ${ }^{2}$, N Wijewardena ${ }^{3}$, H R Senevirathne ${ }^{4}$, D Lokuhetty ${ }^{5}$ \\ Sri Lanka Journal of Diabetes Endocrinology and Metabolism 2011; 1: 37-40
}

\begin{abstract}
Rapid onset of virilization in a post-menopausal woman is usually the result of androgen secretion from a tumour of adrenal or ovarian origin. Androgen secreting neoplasms of the ovary are rare and usually show autonomous secretion. We describe the case of a 61-year-old woman with high serum testosterone and inappropriate normal estrogen despite menopause. Localization of the tumour was not possible by conventional ultrasound or computerized tomography scanning, and was achieved by venous sampling. Complete cure was achieved by oophorectomy. Histopathological examination confirmed the diagnosis. Postoperatively her testosterone returned to normal. Two months after surgery she showed regression of features of hyperandrogenism.
\end{abstract}

\section{Introduction}

Postmenopausal virilization due to an androgen producing ovarian tumour may be autonomous or gonadotrophin driven. Ovarian tumours showing this characteristic include steroid cell tumours of uncertain origin, Leydig cell tumours, granulosa cell tumours, hilus cell tumours and the rare stromal luteoma $(1,2)$. Steroid cell tumours of the ovary account for less than $0.1 \%$ of all ovarian tumours. The subtype, not otherwise specified, is associated with androgenic changes in approximately one half of the patients. Because most of these tumours are diagnosed in an early stage and do not recur or metastasize, little is known about their response to therapies such as chemotherapy or radiation $(1,3)$.

\section{Case report}

A 61-year old woman presented with an 8 month history of increasing hair growth affecting the face, hands, abdomen, chest and back and deepening of voice. She attained menarche at 12 years and had normal menstrual cycles until she attained menopause at the age of 36 years. She had a history of diabetes, hypertension and dyslipidemia. Physical examination revealed her to be virilized with clitoromegaly and increased hair growth in face, hands, abdomen, chest and back. (Modified Ferriman and Gallway score 14/22).

Initial investigations showed an elevated total testosterone (total testosterone of $5.80 \mathrm{ng} / \mathrm{ml}(0.06-0.82)$ ), with normal dihydroepiandrosterone sulphate (DHEAS) (DHEAS 84.6 $\mu \mathrm{g} / \mathrm{dl}$ (35-430)). FSH, LH and estradiol were not compatible with the postmenopausal state. (FSH22.6 U/1 (post menopause >25U/1), LH $8.38 \mathrm{U} / 1$ (post menopause >50/l), Estradiol $32 \mathrm{pg} / \mathrm{ml}(<20-40)$ ). Low dose dexamethasone suppression test (LDDST), did not suppress the total testosterone. (At 48 hours - $14.21 \mathrm{ng} /$ $\mathrm{ml}$ (ND - 0.62)) confirming the non-adrenocorticotrophic hormone dependency of the hyperandrogenic state, thus favouring ovary as the possible source. A pelvic and transvaginal ultrasound examination did not show any abnormalities. The ovaries were of normal size and appearance. ACT scan of the pelvis and adrenal gland did not reveal any abnormalities, giving no clue as to the origin of the hyperandrogenism.

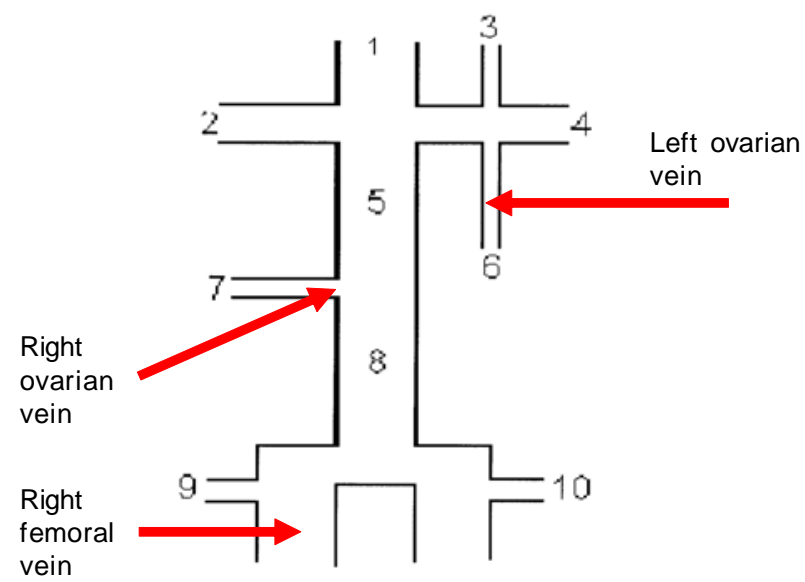

Figure 1. Ovarian vein sampling locations.

${ }^{1}$ Senior Registrar, Endocrinology, ${ }^{2}$ Consultant Endocrinologist, ${ }^{3}$ Consultant Interventional Radiologist, National Hospital of Sri Lanka, ${ }^{4}$ Professor of Obstetrics and Gynaecology, ${ }^{5}$ Professor of Pathology, Faculty of Medicine, University of Colombo, Sri Lanka. 
Ovarian vein sampling was performed. Figure 1 shows the sampling locations and the results are summarized in Table 1. There was a right to left gradient of 1.9 times (higher on right) suggesting the right ovary as the source of testosterone and estradiol production.

Table 1. Summary of the results of ovarian vein catheterization

\begin{tabular}{llll}
\hline & $\begin{array}{l}\text { Right } \\
\text { ovarian } \\
\text { vein }\end{array}$ & $\begin{array}{l}\text { Left } \\
\text { ovarian } \\
\text { vein }\end{array}$ & Ferora \\
\hline Testosterone $(\mathrm{ng} / \mathrm{ml})$ & 18.10 & 10.6 & 10.7 \\
Estradiol $(\mathrm{pg} / \mathrm{ml})$ & 70.0 & 59.0 & 53.0 \\
Cortisol $(\mu \mathrm{g} / \mathrm{dl})$ & 13.7 & 15.3 & 12.4 \\
Testosterone/Cortisol ratio & 1.32 & 0.69 & \\
Testosterone/Estradiol ratio & 0.25 & 0.17 & \\
\hline
\end{tabular}

The patient was referred to the gynecological team. Because of the malignant potential of the steroid cell tumours, in women who have completed child bearing total abdominal hysterectomy and bilateral salpingooophorectomy and complete surgical staging is the preferred treatment while unilateral salpingooophorectomy is reserved for those who desire future fertility (4). Therefore our patient underwent a total abdominal hysterectomy and bilateral salpingooophorectomy despite the venous sampling lateralizing right ovary as the possible source.

The right ovary was $25 \times 15 \times 15 \mathrm{~mm}$ while the left ovary was $35 \times 30 \times 25 \mathrm{~mm}$. Right ovary showed two small nodules of same size measuring $6 \times 6 \times 6 \mathrm{~mm}$. The cut surface showed a well circumscribed yellowish area in the medulla. Microscopic examination showed a well circumscribed tumour composed of sheets, nests and clusters of round cuboidal cells with mildly pleomorpic central vesicular nuclei. The nuclei showed fine granular chromatin and single prominent nucleoli. Mitotic activity was sparse. Some cells showed intranuclear inclusions. The cytoplasm was scanty and acidophilic in some cells and some cells showed abundant foamy cytoplasm with lipid vacuoles. Reinke crystalloids were not identified. Intracytoplasmic liposfuschin pigments were also noted in some cells. The stroma was dense and fibrotic. Some areas showed foci of lymphocytic infiltration. The nodules seen on the ovarian surface showed broad based papillae with a core of fibro ovarian stroma lined by benign cuboidal epithelium. These features were in keeping with an ovarian steroid cell tumour, not otherwise specified (NOS). The uterus showed non secretory endometrium with cystic atrophy. The left ovary was normal.

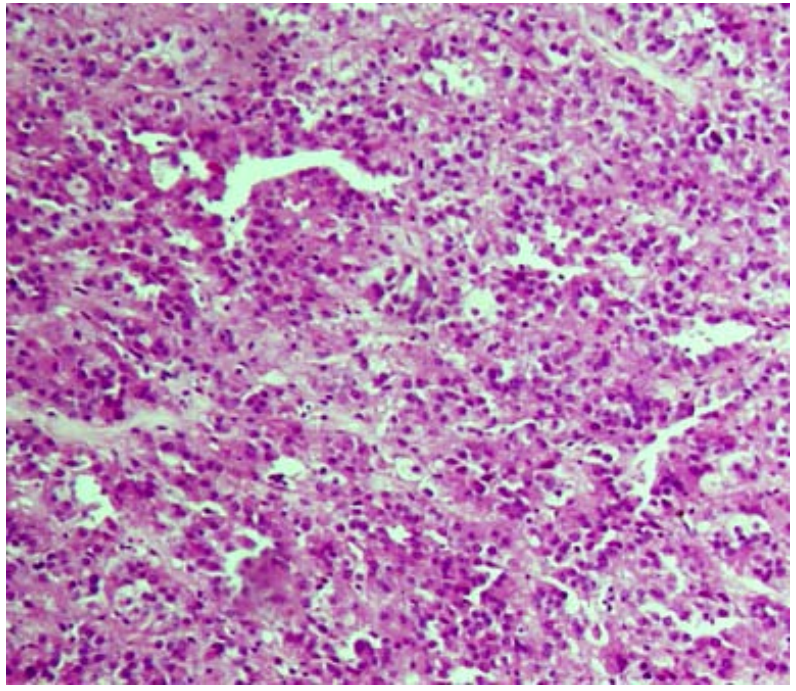

A

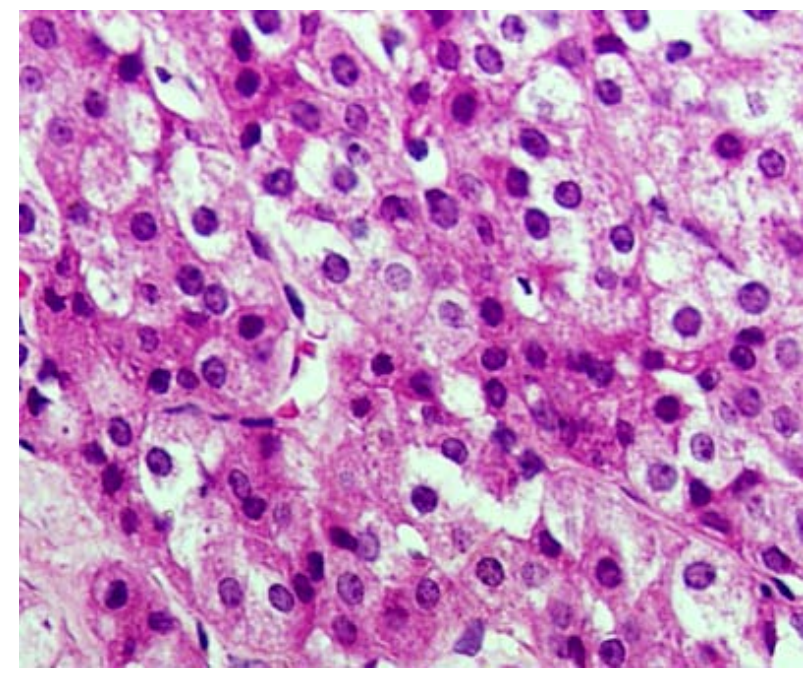

B

Figure 2. Histology of the right ovary, A - in low power field and $B$ - in high power field show clusters of round cuboidal cells with mildly pleomorpic central vesicular nuclei with single prominent nucleoli.

Two weeks after surgery her testosterone returned to normal. Two months after surgery she showed regression of excess hair growth, and reduction in clitoromegaly. The patient is being followed up regularly with measurement of testosterone levels as marker of recurrence.

\section{Discussion}

Both ovarian and adrenal tumours may cause hyperandrogenism in women. While clinical history and baseline serum androgens (testosterone, androstenedione, and DHEAS) may increase clinical suspicion, the localization of virilizing tumours may require several 
diagnostic modalities, including dynamic ovarian and adrenal assessment, and diagnostic imaging. In contrast to ovarian tumours, adrenal tumours are generally identified easily by imaging techniques. As a result, an ovarian source may be suspected when anatomic studies of the adrenal glands are normal. Selective venous catheterization has been employed to aid in the diagnosis and localization of androgen-secreting ovarian and adrenal tumours $(1,2)$.

We describe a case of a postmenopausal woman with recent onset of rapidly progressive virilization. Her initial investigations confirmed hyperandrogenism. Normal DHEAS levels and non suppressible total testosterone during a low dose dexamethasone suppression test favoured ovary and not adrenals as the possible source. Imaging modalities didn't give a clue regarding the source of hyperandrogenism. Selective venous catheterization of ovarian vessels was arranged and based on findings on selective ovarian vein sampling our patient was diagnosed with an androgen secreting tumor localized to the right ovary. As demonstrated by venous sampling, this tumour not only secreted testosterone but also estradiol. Histology of the tumour was proved to be a steroid cell tumour of the ovary.

The spectrum of ovarian neoplasms covers an extremely wide range of tumours. The best recognized of these are the surface epithelial cell tumours. Amongst the less common variants, lipid or steroid cell tumours comprise an important category, although they account for only $0.1 \%$ of all ovarian tumours (2).

The term steroid cell tumours not otherwise specified (NOS) was first described by Scully and signifies that the cell lineage is not defined, and they cannot be categorized as either stromal luteomas or leydig cell tumours. However, the majority of cases fall under this category $(3,5)$.

Steroid cell tumours, NOS are associated with androgenic changes in 50\% of the cases, oestrogenic in 6 to $23 \%$ cases while few cases have shown progestational changes. Our patient had an increase in both testosterone and estradiol. About 10 to $15 \%$ of the patients are asymptomatic, with tumours detected incidentally during routine pelvic examination or in a surgical intervention.

Interestingly our patient has attained menopause at the age of 36 years but she had developed signs and symptoms of virilization only 25 years later (At the age of 61). We were interested to find out whether there can be any association between the onset of menopause and virilization. Although there are reported cases of premature menopause and virilization we couldn't find cases which had longer interval between the onset of premature menopause and virilization as in our case.

The small size (usually $<1 \mathrm{~cm}$ ) accounts for the poor visualization with ultrasound and CT scanning. Selective venous catheterization has been employed to aid in the diagnosis and localization of androgen-producing lesion when the imaging modalities have failed. Based on a series of seven patients, Moltz and colleagues reported that an ovarian gradient of $>2.7$ correctly identified the location of tumor. It was found that most of the tumours were found on the right side and the venous sampling worked better to identify right sided tumours $(3,5)$. In line with previous studies, selective venous sampling in our case proved to be highly effective in tumour localization with a marked testosterone gradient (gradient of 1.9) being present in the right ovarian vein. Venous sampling is an invasive procedure which is operator dependent and is also dependent on reliable anatomy because differences in catheterization technique and placement may influence the results $(6,7)$.

The primary treatment is surgical removal of the primary lesion. In a young patient, unilateral salpingooopherectomy is adequate since the frequency of bilaterality is only $6 \%$, but follow-up is essential and should include measurement of hormone levels as a marker for recurrence. In older patients, hysterectomy and bilateral salpingo-oopherectomy with surgical staging and bulk removal of tumor is recommended $(4,5)$. In our patient too considering the malignant potential favoured procedure was hysterectomy and bilateral salpingo-oopherectomy.

There are no reports of effective radiation or chemotherapy. The main reason for poor understanding of the therapeutic value of chemotherapy and radiotherapy in the treatment of these tumours is due to the rarity of the tumour $(4,8)$.

The most important factor to be determined in lipid/ steroid cell tumours of the ovary is whether the tumour has malignant features or not. In one of the major studies done on lipid cell tumours of the ovary, certain histopathological findings were found to correlate highly with clinically malignant behaviour. These can be summarized as 2 or more mitotic figures per 10 high power fields (92\%), necrosis (86\%), a diameter $\geq 7 \mathrm{cms}(78 \%)$, haemorrhage $(77 \%)$, and grade $2-3$ atypia $(64 \%)(4,8)$.

It is interesting to note that in our case, the microscopic appearance did not reveal any prominent findings in favour of malignancy. Mitotic activity was sparse, there was no haemorrhage or necrosis, tumour size was less than $7 \mathrm{cms}$ in all dimensions.

It is also known that pathologically benign steroid cell tumours can behave in a clinically malignant fashion. Therefore, careful follow-up is essential in such cases which do not have clinical or pathological evidence of malignancy. As such, presence of metastasis may be the only definite evidence of malignant behavior (8). Our patient needs regular follow-up to look for recurrence. 


\section{Conclusion}

The present case demonstrates diagnostic and therapeutic challenge posed by androgen-secreting ovarian tumors. We report this case to highlight how venous sampling helped to resolve the source of androgen production. When diagnostic uncertainty exists, selective venous sampling may be useful to localize a tumour, and thereby help in the definitive management of the patient.

\section{References}

1. Levens ED, Whitcomb BW, John M. Csokmay, Nieman LK. Selective venous sampling for androgen producing ovarian pathology. Clinical Endocrinology 2009; 70(4): 606-14.

2. Haji AG, Sharma S, Babu M,Vijaykumar DK, Chitrathara $\mathrm{K}$. Androgen secreting steroid cell tumor of the ovary in a young lactating women with acute onset of severe hyperandrogenism: a case report and review of literature. $J$ Med Case Reports 2007; 1: 182.

3. Stephens JW, Katz JR, McDermott N, MacLeanand AB
Bouloux PMG. An unusual steroid-producing ovarian tumour: case report. Hum Reprod 2002; 17(6): 1468-71.

4. Powell JL, Dulaney DP, Shiro BC. Androgen-Secreting Steroid Cell Tumor of the Ovary. South Med J 2000; 93: 12.

5. Moltz L, Pickartz H, Sorensen R, Schwartz U, Hammerstein J. Ovarian and adrenal vein steroids in seven patients with androgen-secreting ovarian neoplasms: selective catheterization findings. Fertility and Sterility 1984; 42: 585-93.

6. Kaltsas GA, Mukherjee J, Kola B, et al. Is ovarian and adrenal venous catheterization and sampling helpful in the investigation of hyperandrogenic women. Clinical Endocrinology 2003; 59: 34-43.

7. Mok J, Sohn W. Surgical management of steroid cell tumors of the ovary. Journal of Gynecologic Oncology 2003; 8: 173-8.

8. Mehdi G, Ansari HA, Sherwani RK, Rahman K, Akhtar N, Ovarian Steroid Cell Tumour: Correlation of Histopathology with Clinicopathologic Features. Pathology Research International 2011, Article ID 987895, 5 pages doi:10.4061/2011/987895 8 www.hindawi.com/journals/ pri/2011/987895/ (Accessed on 17th May 2011). 